\title{
The influence of metal/perovskite-type oxide interfaces on tunability of thin film capacitors
}

\author{
Naohiro HORIUCHI, ${ }^{\dagger}$ Takuya HOSHINA, Hiroaki TAKEDA and Takaaki TSURUMI
}

The Graduate School of Science and Engineering, Tokyo Institute of Technology, Ookayama, Meguro, Tokyo $152-8552$

\begin{abstract}
We propose a calculation model that can predict the influence of interface on capacitance-voltage characteristics of metalinsulator-metal type thin film capacitor with perovskite type oxide. The simulation could successfully describe the results of capacitance-voltage measurements of the barium strontium titanate thin film capacitors with top electrodes of Pt, Au, and Ag. It is found that the electrode dependent on the tunability is derived from the work function of the electrode metal. The simulation model is base on the Schottky model that can be employed to explain the dielectric properties of metal/perovskite type oxide junction. The modified Schottky model considers the electric field dependence of permittivity and the back flow of electrons from metal to defect states located in the band gap of the perovskite oxide. This flow forms negatively charged space at the interface and affects the electric properties of the thin film capacitors.
\end{abstract}

(C2010 The Ceramic Society of Japan. All rights reserved.

Key-words: Schottky junctions, Perovskite-type oxide, Thin-film capacitor, Tunability, Barium strontium titanate, Nb doped Strontium titanate

[Received April 22, 2010; Accepted July 15, 2010]

\section{Introduction}

Perovskite oxide, such as $\mathrm{SrTiO}_{3}, \mathrm{BaTiO}_{3}$ and $(\mathrm{Ba}, \mathrm{Sr}) \mathrm{TiO}_{3}$, are important materials for thin film electronic devices. ${ }^{1), 2)}$ They are used in Metal-insulator-metal (MIM) type thin film capacitor. In such MIM type capacitors, the metal/insulator interface has a strong influence on the properties, e.g. the leakage current behavior, ${ }^{3)}$ cycling stability, ${ }^{4}$ and high frequency dielectric losses ${ }^{5)}$ are influenced. Therefore, selects of metals for electrodes can have a strong impact on the device characteristics. The Schottky model that explains the electric properties of metal/ semiconductor junction is the most famous model that predicts the characteristics of metal/perovskite type oxide junctions.

However, at junctions consisting of metal and perovskite-type oxide, some deviations from the conventional Schottky model are found. For instance, hysteretic current-voltage ( $I-V)$ characteristics have been observed, ${ }^{6)-8)}$ namely the resistance at the junction is changed by an external electric field. The resistive switching can not be depicted by the conventional Schottky model. Moreover, the barrier heights obtained from capacitancevoltage $(C-V)$ characteristics are higher than expected by the Schottky model, giving rise to the deviation from that obtained from $I-V$ characteristics. ${ }^{6)-11)}$ Modification of the Schottky model was required to adapt for metal/perovskite-type oxide junctions.

In MIM type BST thin-film capacitor, apparent permittivity decreased with decreasing BST film thickness due to existences of interface layers having lower permittivity than those of the interior BST films. ${ }^{12-14)}$ The low permittivity layer is formed as below. The Schottky model represents depletion layers are formed at the interface. Electric field is formed in the depletion layer, and depresses the permittivity in the depletion layer, because the permittivity of BST has strong electric field dependence. The capacitances of the BST thin film capacitors are tunable by external bias voltage. The external bias voltage is

Corresponding author: N. Horiuchi; E-mail: horiuchi.n.aa@m. titech.ac.jp also applied across the interfaces, which should change the electric properties of the depletion layer. If the modified Schottky model were established, we could predict the influences on the electric properties of the BST thin-film capacitor.

In this report, we study the influence of the interface on tunability of thin film BST capacitor. First, a modified Schottky model was proposed to describe the electric properties of metal/ perovskite-type oxide junction, by investigation of the electric properties of metal/Nb-doped $\mathrm{SrTiO}_{3}$ junctions. We present the influence of the interfaces on tunability of BST thin-film capacitor. The modified Schottky model considers that electrons flow into the states owing to the energy level difference between the Fermi level of the metal and interface states, which diffuse to depth direction from the interface. The tunability of the BST capacitors have dependence on the electrodes of the capacitors, which is explained by the simulation based on the modified Schottky model.

\section{Experiment}

\subsection{Metal/Nb:STO junction}

$\mathrm{Nb}$-doped $\mathrm{SrTiO}_{3}(100)$ (Nb:STO) was used for experiments as a representative of a perovskite-type oxide semiconductor. Junctions of metal/perovskite-type oxide were fabricated by contacting electrodes to $\mathrm{Nb}: \mathrm{STO}$ single crystal surfaces with doping ratios of $0.05 \mathrm{wt} \%$. $\mathrm{Pt}, \mathrm{Au}, \mathrm{Ag}$, and $\mathrm{SrRuO}_{3}$ (SRO) electrodes were fabricated with an RF magnetron sputtering using a shadow mask on the substrate. The SRO electrodes were deposited at $650^{\circ} \mathrm{C}$. The sputter gas was $\mathrm{Ar}$ and $\mathrm{O}_{2}$ mixture $(\mathrm{Ar} /$ $\mathrm{O}_{2}=4: 1$ ). The other metal electrodes were deposited at $130^{\circ} \mathrm{C}$. Before the depositions, the substrates were annealed at $650^{\circ} \mathrm{C}$ under an oxygen partial pressure of $2 \times 10^{-4}$ Torr for 1 hour in the sputtering system. The Pt, Ag, and SRO were capped with a layer of Au to ensure comparable contact to the probe. InGa alloy electrode was used as the back electrode behind the substrates to form Ohmic contacts to $\mathrm{Cu}$ lead wires. The $I-V$ and $C-V$ characteristics were measured with Keithley 6487 and Agilent 4294A, relatively. 


\subsection{BST thin-film capacitor}

BST thin films were prepared with the RF magnetron sputtering on $\mathrm{SrRuO}_{3}$ (SRO)/ $/ \mathrm{SrTiO}_{3}$ (STO) single crystal substrates. The SRO films were deposited as bottom electrodes using the same sputtering system. The sputter gas was $\mathrm{Ar}$ and $\mathrm{O}_{2}$ mixture $\left(\mathrm{Ar} / \mathrm{O}_{2}=4: 1\right)$. The BST target is stoichiometric $\mathrm{Ba}_{0.5} \mathrm{Sr}_{0.5} \mathrm{TiO}_{3}$. The thicknesses of the BST films were 40, 60, 80 and $120 \mathrm{~nm}$. Top electrodes metals $\mathrm{Pt}, \mathrm{Au}$, and $\mathrm{Ag}$ were deposited on the BST film. Dielectric properties were measured with Agilent 4294A at room temperature.

\section{Theory}

We present the theory for calculations of $C-V$ characteristics of BST thin film capacitors. First, the modified Schottky model for metal/perovskite type oxide is proposed. Second, the calculation method of the $C-V$ characteristics of BST capacitors based on the modified model is described. The basic concept of our modified model consists of two parts. One is that the model incorporates electric field dependence of permittivity in the depletion layer. The other is an assumption of electric flow form metal electrode to defect states located in the band gap of the perovskite type oxide

\subsection{Metal/Nb:STO junction}

In conventional Schottky model, the relation between the capacitances of the junction $\left(1 / C^{2}\right)$ and applied bias voltage is expressed below: ${ }^{15)}$

$$
1 / C^{2}=2\left(V_{\mathrm{bi}}-V\right) / q \varepsilon_{0} \varepsilon_{\mathrm{r}} N_{\mathrm{d}}
$$

where $V_{\mathrm{bi}}$ is the built-in potential, $\varepsilon_{0}$ is the vacuum permittivity, $\varepsilon_{\mathrm{r}}$ is the relative permittivity, and $N_{\mathrm{d}}$ is the donor density. The electric field dependence of permittivity in the depletion layer was neglected in Eq. (2), although the STO and BST have strong electric field dependence. ${ }^{16)}$

The $C-V$ characteristics of junctions are calculated using a Poisson's equation. In order to incorporate the electric field dependence of permittivity into the calculation, the permittivity $\varepsilon_{\mathrm{r}}(x)$, the charge density $\rho(x)$, the electric field $E(x)$, and the potential $V(x)$ are expressed as a function of the distance from the interfaces $x$. The Poisson's equation should be rewritten as below. The electric displacement

$$
D=\varepsilon_{0} \varepsilon_{\mathrm{r}}(x) E(x)=-\varepsilon_{0} \varepsilon_{\mathrm{r}}(x) \frac{\partial V(x)}{\partial x}
$$

is substituted into Gauss's law: $\partial D / \partial x=\rho(x)$. Then, a version of Poisson's equation was obtained as below:

$$
\frac{\partial^{2} V(x)}{\partial x^{2}}=-\frac{\rho(x)}{\varepsilon_{0} \varepsilon_{\mathrm{r}}(x)}-\frac{1}{\varepsilon_{\mathrm{r}}(x)} \frac{\partial V(x)}{\partial x} \frac{\partial \varepsilon_{\mathrm{r}}(x)}{\partial x} .
$$

The permittivity has electric field dependence,

$$
\varepsilon_{\mathrm{r}}(E)=f(E) \text {. }
$$

Here, the Eq. (4) is substituted by the electric field dependence of the permittivity of $\mathrm{Nb}$ :STO given by $\varepsilon_{\mathrm{r}}(E)=b / \sqrt{a+E^{2}}$, where $a$ and $b$ are constants $\left(a=1.64 \times 10^{15} \mathrm{~V}^{2} / \mathrm{m}^{2}\right.$ and $\left.b=1.48 \times 10^{10} \mathrm{~V} / \mathrm{m}\right) .{ }^{16)}$ The profiles of $V(x), E(x), \varepsilon_{\mathrm{r}}(x)$, and the capacitance of the junctions are obtained from the $\rho(x)$ and the boundary conditions by numerical solving Eqs. (3) and (4) using a finite difference method. The boundary conditions are determined from the external bias voltage and the Schottky barrier heights. The Schottky barrier heights $\left(\phi_{\mathrm{B}}{ }^{\mathrm{SL}}\right)$ are calculated from the work functions of metals $\left(\phi_{\mathrm{e}}\right)$ by the Schottky-Mott relationship $\phi_{\mathrm{B}}{ }^{\mathrm{SL}}=\phi_{\mathrm{e}}-\chi$ (the so-called Schottky limit), where (a)

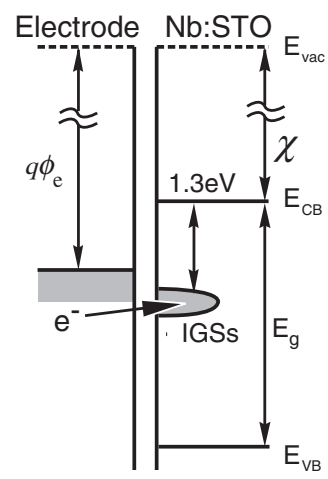

(b)

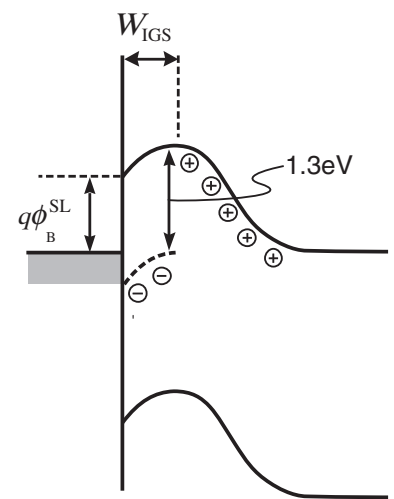

Fig. 1. Schematic band diagram of metal/Nb:STO junction. (a) Before the formation, and (b) after the formation.

$\chi$ is electron affinity of $\mathrm{Nb}: \mathrm{STO}$. As a result, the $C-V$ characteristics are calculated as a function of the work function of metals.

The other modification is an assumption of an electrons flow from the electrode metal to defect states located in the band gap of the perovskite oxide. Photoemission spectroscopy studies have revealed broad states forming in the band gap of electron-doped STO (in-gap states: IGSs). ${ }^{17), 18)}$ The Schottky barrier heights are very sensitive to a preannealing in an oxygen-rich atmosphere, ${ }^{9), 10)}$ indicating that the IGSs are associated with oxygen defects. ${ }^{17)}$ As shown in Fig. 1(a), when the junction was formed, electrons in the metal electrode flow into the IGSs, owing to the energy level difference between the Fermi level of the metal and IGSs. This flow of electrons form negative space charge region in the vicinity of interfaces (Fig. 1(b)). In the modified Schottky model, the capacitance of the junctions is calculated from the carrier density profile $\rho(x)$ involving the negative space charge regions. The $C-V$ characteristics are calculated as a function of the work function of metals.

\subsection{BST tunability}

We calculate the $C-V$ characteristics of the metal/BST/SRO capacitors based on the modified Schottky model describe as above. Figure 2(a) shows the schematic band diagram of the metal/BST junction. The Schematic band diagrams of the BST capacitor is shown in Fig. 2(b). The band bending at BST/SRO junction is negligible because SRO/Nb:STO junction has no negative space charge. The BST band near the interface is bending because of the negative space charge region. Although in the metal/semiconductor junctions, the all external voltage are only applied to the interface, the external voltage are divided into the interface and BST interior layer in the BST thin film capacitor. The Schematic band diagrams of the BST capacitor under the external bias were shown in Fig. 2(c) and (d). The potential profile through the BST film can be obtained by solving Eqs. (3) and (4), provided that the electric field dependence $\varepsilon_{\mathrm{r}}$ of BST was given. The $\varepsilon_{\mathrm{r}}$ is calculated based on the Laudau model as follows: ${ }^{19)}$

$$
\varepsilon_{\mathrm{r}}(E)=\varepsilon_{\mathrm{MAX}}\left\{2 \cosh \left[\frac{2}{3} \sinh ^{-1}\left(\frac{2 E}{E_{2}}\right)\right]-1\right\}^{-1},
$$

where $\varepsilon_{\mathrm{MAX}}$ is the maximum permittivity under no electric field, and $E_{2}$ is the electric field at which $\varepsilon\left(E_{2}\right)=\varepsilon_{\mathrm{MAX}} / 2$. Accordingly, the $C-V$ characteristics can be calculated as a function of 
(a)

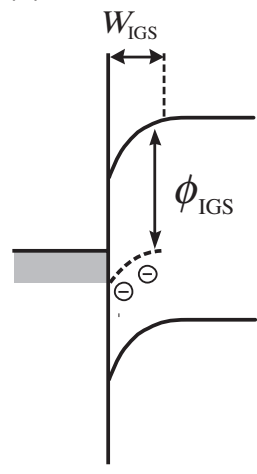

(b)

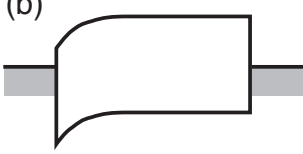

(c)

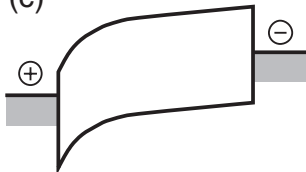

(d)

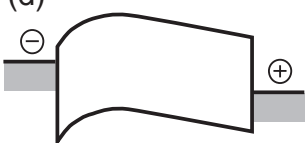

Fig. 2. Schematic band diagram of (a) metal/BST junction, (b), (c), (d) metal/BST/SRO capacitors.

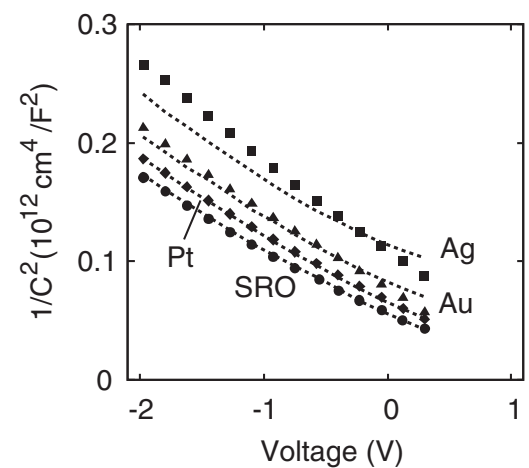

Fig. 3. The $C-V$ characteristics of the junction between the various electrodes and $0.05 \mathrm{wt} \%-\mathrm{Nb}: \mathrm{STO}$. The marks and lines denote measured and calculated results.

the work function of metals in the same way as the metal/ $\mathrm{Nb}$ :STO junction.

\section{Results and discussion}

\subsection{Metal/Nb:STO junction}

Figure 3 shows the $C-V$ characteristics: the relationship between $1 / C^{2}$ and bias voltage of metal/Nb-STO interfaces measured at $10 \mathrm{kHz}$. The bias voltage was swept from negative to positive. Marks in the figure denote experiment results and lines denote calculation results that will be discussed later. The frequency dependence of the capacitances and hysteresis in the $C-V$ characteristics were negligible.

We simulated for the $C-V$ characteristics based on the modified Schottky model. The barrier heights used in the simulation $\left(\phi_{\mathrm{B}}{ }^{\mathrm{SL}}\right)$ were listed in Table 1 . The calculated $C-V$ characteristics are shown in Fig. 3. The widths of negative space charge regions $W_{\text {IGS }}$ listed in Table 1 were obtained as results of fitting to the $C$ $V$ characteristics in Fig. 3. The carrier density of $1.6 \times 10^{19} \mathrm{~cm}^{-3}$ was also obtained, which is comparable to previously reported values of $1.1 \times 10^{19} \mathrm{~cm}^{-3} .^{23)}$ As shown Table 1, the electrode with smaller work function has the larger $W_{\mathrm{IGS}}$, because the difference between the level of IGSs and the Fermi level of metal is increase with decreasing the work function. The position of the IGSs level for each electrode was evaluated from $W_{\text {IGs. The IGSs }}$ level were approximately located at $1.3 \mathrm{eV}$ blow the conduction band of Nb:STO, which is independent on the electrode. Consequently, the modified Schottky model could well describe the $C-V$ characteristics of metal/Nb:STO junction.

Table 1. The parameters determined for each top electrode, where $q \phi_{\mathrm{e}}$ is the work function of electrodes. The values in parenthesis are used for the simulations and calculations of the $\phi_{\mathrm{B}}{ }^{\mathrm{SL}}\left(\phi_{\mathrm{B}}{ }^{\mathrm{SL}}=\phi_{\mathrm{e}}-\chi\right) . \chi$ is the electron affinity of $3.9 \mathrm{eV}$ in $\mathrm{Nb}: \mathrm{STO}^{20)}$

\begin{tabular}{lccccc}
\hline & $\begin{array}{c}q \phi_{\mathrm{e}}{ }^{21), 22} \\
(\mathrm{eV})\end{array}$ & & $\begin{array}{c}\phi_{\mathrm{B}}{ }^{\mathrm{SL}} \\
(\mathrm{V})\end{array}$ & $\begin{array}{c}W_{\mathrm{IGS}} \\
(\mathrm{nm})\end{array}$ & $\begin{array}{c}\phi_{\mathrm{B}}{ }^{\mathrm{CV}} \\
(\mathrm{V})\end{array}$ \\
\hline $\mathrm{SRO}$ & 5.2 & $(5.2)$ & 1.3 & 0 & 1.4 \\
$\mathrm{Pt}$ & $5.12-5.98$ & $(5.3)$ & 1.4 & 10.1 & 1.6 \\
$\mathrm{Au}$ & $5.1-5.47$ & $(5.1)$ & 1.2 & 19.2 & 1.9 \\
$\mathrm{Ag}$ & $4.52-4.76$ & $(4.7)$ & 0.8 & 38.1 & 2.5 \\
\hline
\end{tabular}

The calculation results well described the measurements in Fig. 3. The slight differences between the calculations and the measurements were derived from simplifications of the calculation model. The charge density of IGSs was assumed to be $1.0 \times 10^{20} \mathrm{~cm}^{-3}$ and independent on the depth from interfaces while actual charge density varies with the depth. ${ }^{15)}$

In order to confirm the contribution of the negative space charge regions, we also calculated for the $C-V$ characteristics based on the modified model without the electron flows into IGSs. The widths of the negative space charge region $\left(W_{\mathrm{IGS}}\right)$ are set to zero. As results of fitting to measurements in Fig. 3, the Schottky barrier height $\left(\phi_{\mathrm{B}}{ }^{\mathrm{CV}}\right)$ listed in Table 1 were obtained. The $\phi_{\mathrm{B}}{ }^{\mathrm{CV}}$ of SRO $(1.40 \mathrm{~V})$ is comparable to the Schottky barrier heights $\left(\phi_{\mathrm{B}}{ }^{\mathrm{SL}}\right)$ predicted from Schottky-Mott relationship. On the contrary, the order of the Schottky barrier height $\left(\phi_{\mathrm{B}}{ }^{\mathrm{CV}}\right)$ obtained was discrepant to that predicted by the Schottky-Mott relationship $\left(\phi_{\mathrm{B}}^{\mathrm{SL}}\right)$. The model without considering the IGSs could not explain the results of $C-V$ measurement. The fact indicate that the electron flow into IGSs and formation of negative space charge regions are essential factor in the junction of metal/ $\mathrm{Nb}: \mathrm{STO}$ junctions.

\subsection{BST tunability}

Marks in Fig. 4 shows the $C-V$ characteristics of the BST thin film capacitor measured at $10 \mathrm{kHz}$. The capacitances in longitudinal axes are normalized by dividing by the capacitances at zero bias. The peaks are deviated form zero voltage and shifted to negative direction, where the positive bias was defined that the top electrodes were positively biased for the bottom electrodes. The deviations of the peak positions become larger in order of $\mathrm{Pt}$, $\mathrm{Au}$ and $\mathrm{Ag}$. Figure 5 shows tunability as a function of the BST film thickness. Tunability $n$ is defined by $n=(C(0)-C(V)) /$ $C(0)$, where $C(0)$ and $C(V)$ are the capacitances at zero and $V$ of bias voltage, respectively. The $V$ of bias voltage is the voltage corresponding to electric field $100 \mathrm{MV} / \mathrm{m}$. Tunability increases with increasing film thickness in each kind of metal electrodes. In addition, the tunability becomes lower in order of Pt, Au and Ag. Such electrode dependences of the peak position and tunability were associated with the widths of the negative space charge region $\left(W_{\mathrm{IGS}}\right)$. The deviations of the peak position increased with increasing the $W_{\mathrm{IGS}}$.

We calculated the $C-V$ characteristics of the metal/BST/SRO capacitor including the interface effect based on the modified Schottky model. The calculated $C-V$ curves and tunability are shown as lines in Fig. 4 and Fig. 5, respectively. They well agree with the measurements. The electric field dependence of the BST thin film permittivity is obtained as parameters in Eq. (5), $\varepsilon_{\mathrm{MAX}}=250$ and $E_{2}=30 \mathrm{MV} / \mathrm{m}$. As a result of the calculation, the energy differences between the IGSs level and the Fermi level of metals are obtained as follows: these of $\mathrm{Pt}, \mathrm{Au}$, and $\mathrm{Ag}$ are $1.15,1.20$, and $1.58 \mathrm{~V}$, respectively. These values are predicted 
(a) $60 \mathrm{~nm}$

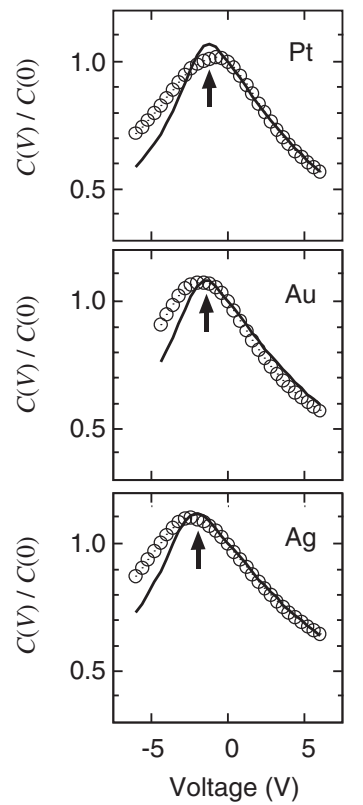

(b) $120 \mathrm{~nm}$

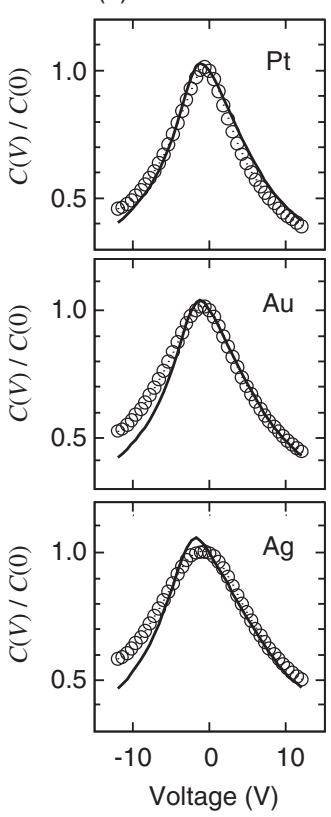

Fig. 4. The $C-V$ characteristics of the BST thin film capacitor with thickness of $60 \mathrm{~nm}$ and $120 \mathrm{~nm}$. Marks denote the measurement results and lines denote the calculation results.

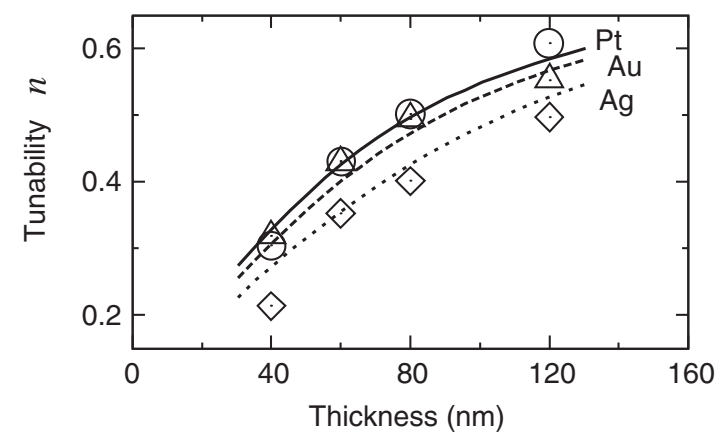

Fig. 5. Tunability $n$ as a function of BST thin film thickness. Marks denote the measurement results and lines denote the calculation results.

from the work function of electrode, assuming that the IGSs level is located at $2.2 \mathrm{~V}$ below the conduction band of the BST. The position of IGSs level in the BST films is different from that in the $\mathrm{Nb}$ :STO. It is considered that the origin of the IGSs in the BST films is oxygen defect and composition deviation from stoichiometry which was generated in the deposition process with sputtering. Therefore, the position of IGSs level in the BST films are different from that in $\mathrm{Nb}$ :STO single crystal. Accordingly, we can conclude that the electrode dependence of the $C-V$ characteristics was explained in terms of the work function of metal using the model.

In Fig. 6, we show a calculation result of depth profiles of potential, electric field, and the permittivity for the BST capacitor of $40 \mathrm{~nm}$ film thickness as a representative. The profiles were obtained through the calculation of the tunabilities in Fig. 5, so the parameters used in the simulation is the same as mentioned below. The depth profiles are shown only in neighborhood of the electrodes. The depth $0 \mathrm{~nm}$ denote the interface of metal and BST. The negative space charge region is from $0 \mathrm{~nm}$ to about $7 \mathrm{~nm}$. In the vicinity of the interface, the strong electric field exists, and the permittivity is strongly depressed by the electric

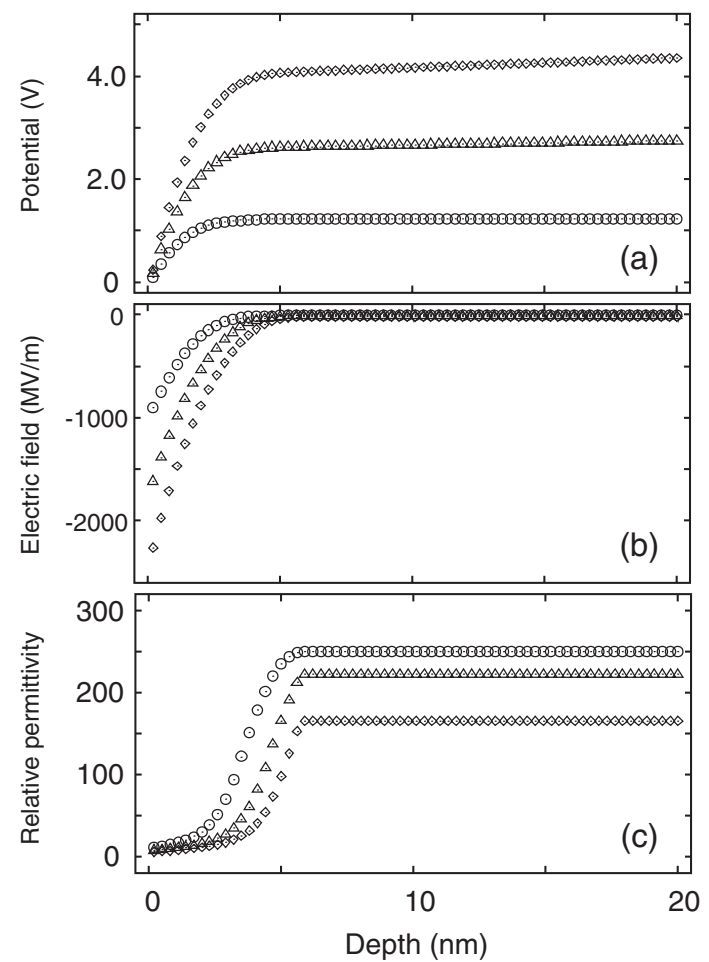

Fig. 6. The depth profiles of (a) potential, (b) electric field, and (c) permittivity of the thin film capacitor with film thickness of $40 \mathrm{~nm}$ under bias voltage $0 \mathrm{~V}(\bigcirc), 2 \mathrm{~V}(\nabla), 4 \mathrm{~V}(\diamond)$.

field. The electric field is derived from the negative space charge regions. As shown in Fig. 6, the voltage applied to the film was divided into the negative space charge region (under $7 \mathrm{~nm}$ ) and the internal BST layer, and a large part of voltage is applied to the negative space charge region. Thus, electric field in internal BST layer becomes smaller. Accordingly, it the negative space charge regions decrease the tunability of the BST thin film capacitor.

\section{Conclusion}

The study proposes a modified Schottky model suitable for metal/perovskite-type oxide junctions. The tunability of barium strontium titanate (BST) thin film capacitors were simulated based on the modified Schottky model. The simulation well described the electrode dependence of the tunability of the BST thin film capacitors with top electrodes of Pt, Au, and Ag. The model considers the formation of negative space charge region that affect on the tunability of the capacitors. The space charge region was formed by electrons flow from metal electrode to the defect states located in the band gap of the perovskite oxide. The flow is due to the energy level difference between the Fermi level of the metal and defect states. Therefore, the space charge region is depending on the work function of electrode metal. That means that, in this model, the electrode dependence of the capacitancevoltage characteristics are simulated from the work functions of metal electrode. Thus, our calculation model for the electric properties of metal/perovskite type oxide junction is useful for designs of dielectric devices.

\section{Reference}

1) N. Setter, D. Damjanovic, L. Eng, G. Fox, S. Gevorgian, S. Hong, A. Kingon, H. Kohlstedt, N. Y. Park, G. B. Stephenson, I. Stolitchnov, A. K. Taganstev, D. V. Taylor, T. Yamada and S. 
Streiffer, J. Appl. Phys., 100, 051606 (2006).

2) M. Dawber, K. M. Rabe and J. F. Scott, Rev. Mod. Phys., 77, 1083-1130 (2005).

3) J. D. Baniecki, R. B. Laibowitz, T. M. Shaw, K. L. Saenger, P. R. Duncombe, C. Cabral, D. E. Kotecki, H. Shen, J. Lian and Q. Y. Ma, J. Eur. Ceram. Soc., 19, 1457-1461 (1999).

4) R. Plonka, R. Dittmann, N. A. Pertsev, E. Vasco and R. Waser, Appl. Phys. Lett., 86, 202908 (2005).

5) O. Auciello, S. Saha, D. Y. Kaufman, S. K. Streiffer, W. Fan, B. Kabius, J. Im and P. Baumann, J. Electroceram., 12, 119131 (2004).

6) R. Waser, R. Dittmann, G. Staikov and K. Szot, Adv. Mater., 21, 2632-2663 (2009).

7) C. Park, Y. Seo, J. Jung and D.-W. Kim, J. Appl. Phys., 103, 054106 (2008).

8) D. Seong, D. Lee, M. Pyun, J. Yoon and H. Hwang, Jpn. J. Appl. Phys., 12, 8749-8751 (2008).

9) R. Schafranek, S. Payan, M. Maglione and A. Klein, Phys. Rev. $B, 77,195310$ (2008).

10) T. Shimizu, N. Gotoh, N. Shinozaki and H. Okushi, Appl. Surf. Sci., 117/118, 400-405 (1997).

11) T. Shimizua and H. Okushi, J. Appl. Phys., 85, 7244-7251 (1999).
12) S. Boesch, J. Son, J. M. LeBeau, J. Cagnon and S. Stemmer, Appl. Phys. Express, 1, 091602 (2008).

13) N. H. Finstorm, J. Cagnon and S. Stemmer, J. Appl. Phys., 101, 034109 (2007).

14) M. Stengel and N. A. Spaldin, Nature, 443, 679-682 (2006).

15) S. M. Sze, "Physics of Semiconductor Devices," 2nd ed., Wiley, New York (1981).

16) T. Yamamoto, S. Suzuki, K. Kawaguchi and K. Takahashi, Jpn. J. Appl. Phys., 37, 4737-4746 (1998).

17) Y. Aiura, I. Hase, H. Bando, T. Yasue, T. Saitoh and D. S. Dessau Surf. Sci., 515, 61-74 (2002).

18) Y. Ishida, R. Eguchi, M. Matsunami, K. Horiba, M. Taguchi, A. Chainani, Y. Senba, H. Ohashi, H. Ohta and S. Shin, Phys. Rev. Lett., 100, 056401 (2008).

19) D. R. Chase, L. Y. Chen and R. A. York, IEEE Trans. Microwave Theory Tech., 53, 3215-3220 (2005).

20) J. Robertson, J. Vac. Sci. Technol. B, 18, 1785-1791 (2000).

21) D. R. Lide, "CRC Handbook of Chemistry and Physics," 86th ed., CRC Press, Boca Raton (2005).

22) M. Minohara, I. Ohkubo, H. Kumigashira and M. Oshima, Appl. Phys. Lett., 90, 132123 (2007).

23) T. Fujii, M. Kawasaki, A. Sawa, Y. Kawazoe, H. Akoh and Y. Tokura, Phys. Rev. B, 75, 165101 (2007). 